Revue d'histoire de l'Amérique française

REVUE D.HISTOIRE DE L'AMÉRIQUE FRANÇAISE

\title{
La bourgeoisie et les mariages, 1729-1748
}

\section{Cameron Nish}

Volume 19, numéro 4, mars 1966

URI : https://id.erudit.org/iderudit/302515ar

DOI : https://doi.org/10.7202/302515ar

Aller au sommaire du numéro

Éditeur(s)

Institut d'histoire de l'Amérique française

ISSN

0035-2357 (imprimé)

1492-1383 (numérique)

Découvrir la revue

Citer cet article

Nish, C. (1966). La bourgeoisie et les mariages, 1729-1748. Revue d'histoire de l'Amérique française, 19(4), 585-605. https://doi.org/10.7202/302515ar d'utilisation que vous pouvez consulter en ligne.

https://apropos.erudit.org/fr/usagers/politique-dutilisation/ 


\section{LA BOURGEOISIE ET LES MARIAGES ${ }^{1}$}

\section{9-1748}

En Nouvelle-France aussi bien que dans les colonies anglaises d'Amérique, comme nous le verrons, le mariage remplissait plusieurs fins outre celles que nous associons généralement à l'émotivité ou à la croissance du genre humain. L'intendant Hocquart reconnut très clairement une de ces fins lorsqu'il donna à Varin la permission d'épouser la fille de Beaujeu: "... cet établissement fait honneur au Sr. Varin et l'attachera particulièrement au service du Roi en cette Colonie...". ${ }^{2}$ Les mariages servaient aussi les intérêts des marchands et de la classe qu'on a appelée aristocratique, même si ces buts n'ont pas été clairement énoncés. Le mariage était un moyen d'atteindre à un rang social et servait d'entrée dans le monde de la richesse. L'argent mariait le rang et le rang mariait l'argent. Dans certains cas, celui d'Henry Hiché, par exemple, le rang a marié la terre. ${ }^{3}$ Sans doute, dans une petite colonie comme l'était la Nouvelle-France, devait-on s'attendre à des liens maritaux très complexes ? Mais les dimensions d'une colonie étaient peut-être sans rapport avec ce phénomène. Cette dernière supposition nous paraît plus juste quand nous examinons les colonies américaines du sud, beaucoup plus nombreuses, et que nous y voyons les mêmes caractéristiques au sujet du mariage.

Frederick B. Tolles, dans son ouvrage sur les marchands de la Pennsylvanie, remarque que les groupes dominants de la

1 Cette monographie est extraite d'une étude plus vaste qui s'intitule La bourgeoisie canadienne, 1729-1748. Le deuxième chapitre de ce travail a été publié. Voir Cameron Nish, "Une bourgeoisie coloniale en NouvelleFrance: Une hypothèse de travail", Actualité économique, vol. 39 , no, 2, (juillet-septembre 1963) : 240-265.

2 "Hocquart au Ministre, 16 octobre 1733", APC, C 11 A, 120-1, Domaine d'Occident, $\mathrm{f} 40$; et R.P.L. Le Jeune, Dictionnaire général de biographie, Histoire, Littérature..., (Université d'Ottawa, 1931), II : 757-758.

3 Voir plus bas sur Hiché. 
vie politique, économique et sociale se mariaient entre eux. ${ }^{4}$ Robert East, dans sa monographie sur les marchands des colonies anglo-américaines, écrit que "par le mariage et d'autres liens de famille, les intérêts commerciaux, les intérêts des propriétaires et ceux des fonctionnaires étaient étroitement liés". 5

Selon Virginia Harrington, la société de la colonie de New York, "... formed a single, privileged ruling class as against the rank and file of small freeholders, tenant-farmers, shopkeepers, artisans and laborers. Generations of inter-marriage had welded... a large interrelated clan whose interests could not be far separated." $\quad$ L. W. Labaree en décrit tous les traits dans le passage qui suit: ${ }^{7}$

Some of the leaders could point with pride to an aristocratic background or connection in England as an explanation of their nosition in America; others owed their rise to thrift, hard work, and foresight, or to sheer native ability; still others had taken advantage of opportunities to acquire land or other forms of wealth by methods that do not always bear too close inspection. Wathever the origin of such men's position, however they came to the top, they tried to stay there themselves and to pass on to their children the standing in the colonial society which they had acquired.

Very soon their came into being groups of leading families, made up of men of wealth and position, their children, and their children's children. It was inevitable that such families should intermarry, and, so they did not just occasionally, but repeatedly, until in some cases, notably in Virginia and

4 Frederick B. Tolles, Meeting House and Counting House: The Quaker Merchants of Colonial Philadelphia, 1682-1763 (New York: W. W. Norton Company Inc., 1963), 62.

5 Robert A. East, "The Business Entrepreneur in a Changing Colonial Economy", Journal of Economic History: The Task of the Economic History, Supplement VI, (1946) : 19.

6 Virginia D. Harrington, The New York Merchants on the Eve of the Revolution (New York: Columbia University Press, 1935), 10.

7 L. W. Labaree, Conservatism in Early America (Ithaca: Cornell University Press, 1959), 2-3. 
New York, their geneological trees became veritable jungles of interwoven branches... the chief merchants ... and the leading lawyers and other professional men usually became affiliated with the dominant group.

Examinons maintenant la Nouvelle-France.

Louis Liénard, sieur de Beaujeu (1683-1750), était un militaire, commandant d'un fort, administrateur et seigneur ${ }^{8}$ tout comme l'était son fils Louis Liénard de Beaujeu, sieur de Villemomble (1716-1802). Le sieur de Villemomble a épousé Louise-Charlotte Cugnet, la fille de François-Etienne Cugnet. ${ }^{9}$ Charlotte Liénard de Beaujeu, la sœur du sieur de Villemomble, a épousé Jean-Victor Varin de la Marre, l'administrateur à Montréal. ${ }^{10}$ Daniel-Hyacinthe-Marie de Beaujeu (1711-1755) était marié à Michelle-Élisabeth Foucault, ${ }^{11}$ la fille de François Foucault le garde-magasin, seigneur, marchand et membre du Conseil Supérieur. ${ }^{12} \mathrm{Ce}$ sieur de Beaujeu est parfois nommé Daniel Liénard de Beaujeu fils. Un de ses enfants était entré par mariage dans la famille Saint-Ange Charly et ainsi Daniel était le beau-père de François-Xavier Charly. ${ }^{13}$ Les Charly étaient des marchands importants et des trafiquants de fourrures.

Maurice Blondeau, d'abord intéressé au commerce intérieur, était allié à plusieurs familles engagées dans l'armée et les postes de traite de l'intérieur, et dans la propriété terrienne en Nouvelle-France. Sa fille, Suzanne, était mariée à Joseph Hertel

${ }^{8}$ Le Jeune, I: 136-137.

${ }^{9}$ Le Jeune, I: 453. Voir aussi la section sur Cugnet, plus bas, et l'appendice à la fin de cet essai sur le sens des liens de parenté.

10 "Hocquart au Ministre, 16 octobre 1733", $A P C, \mathrm{C} 11 \mathrm{~A}$, vol. 120-1, Domaine d'Occident, f 39-40. Aussi Le Jeune, I: 137.

11 Le Jeune, I: 137-138.

12 Le Jeune, I: 642-643.

13 P.-G. Roy, Inventaire des concessions en fief et seigneurie, fois et hommage et aveus et denombrements conservés aux archives de la province de Québec (Québec: Beauceville, 1927), IV: 265. (Désormais cité sous le nom Roy, Seigneurie). Voir aussi la section sur Charly, plus bas. 
de Pierreville. ${ }^{14}$ Un autre enfant, Apolline, avait épousé LouisRené Godefroy de Linctot, ${ }^{15}$ tandis qu'une troisième, MarieMadeleine avait pour mari Pierre-Joseph de Céloron de Blainville. ${ }^{16}$

La "jungle" dont a parlé Labaree ne trouve pas d'illustration meilleure que dans la famille des Boucher. Un mémoire attribué à Gilles Hocquart décrit ainsi la famille des Boucher: ${ }^{17}$

Cette famille est établie à Boucherville près de Montréal, l'ancêtre, qui a près de 90 ans, a plus de 150 enfants, petits-enfants, frères, neveux et petits-neveux.

Nous donnons des exemples seulement pour la période qui va de 1729 à 1748. Joseph Boucher de la Broquerie était marié à Marie-Clémence Gamelin-Maugras. ${ }^{18}$ Les Maugras constituaient un groupe commercial très important et détenaient tout aussi bien de vastes domaines terriens. Jeanne Boucher était mariée à Charles de Sabrevois (1699-1774). Ce dernier était le frère de Clément de Sabrevois, sieur de Bleury (1702-1781), mieux connu, qui était négociant, marchand de bois, seigneur et qui jouissait du monopole du transport à l'intérieur de la colonie. ${ }^{19}$ LaVérendrye, trafiquant et explorateur, était aussi allié aux Boucher. Sa mère était la fille de Pierre Boucher, l'ancien gouverneur des Trois-Rivières. ${ }^{20}$ Pierre Boucher de Boucherville avait pour épouse Anne-Marguerite, fille de Pierre

14 P.-G. Roy, Inventaire des greffes de notaires du régime français (Québec: Archives de la Province de Québec, 1952), Guillet de Chaumont, XVI : 146-147. (Désormais cité sous Roy, Greffes).

15 Le Jeune, II : 156.

16 Claude de Bonnault, "Le Canada Militaire", RAPQ (1949-1951):

425 et Le Jeune, I: 333 . Voir aussi la section sur Linctot, plus bas.

17 "Mémoire au Ministre, 8 novembre 1737".

18 Roy, Seigneurie.

19 P.-G. Roy, Les officiers d'état-major des gouvernements de Québec, Montréal et Trois-Rivières sous le régime français (Lévis, 1919), 277. (Cité désormais sous le titre Roy, Officiers).

${ }^{20}$ L'abbé A Gosselin, L'Eglise du Canada depuis Monseigneur Laval jusqu'à la conquête. Deuxième partie: Mgr Mornay, Mgr Dosquet, Mgr de Lauberivière (4 vol., Québec: Laflamme \& Proulx, 1912), II: 241, et Roy, Officiers, 235 . Voir aussi la section sur La Vérendrye, plus bas. 
Raimbault, marchand, seigneur et administrateur à Montréal. ${ }^{21}$ Un fils de Pierre Boucher, le patriarche de la famille, Jean Boucher, sieur de Montbrun, avait choisi son épouse dans le clan Charest. ${ }^{22}$ Charlotte-Ursule, la fille de Pierre Boucher, sieur de Niverville, s'était mariée à Jean-Baptiste Neveu, de la famille des Neveu, marchands, trafiquants de fourrures et seigneurs. ${ }^{23}$

Les Boucher, de par leur nombre, représentaient toute une trame de relations. Et on peut dire encore que la famille des Boucher, détenant des emplois publics et des terres selon le régime seigneurial, était aussi alliée par le mariage à tous les groupes commerciaux et administratifs importants. Dépassant les environs de Boucherville, elle avait des liens personnels et maritaux qui s'étendaient à toute la colonie.

Le 20 octobre 1742, il y eut un double mariage en NouvelleFrance. Étienne Charest, membre de la bourgeoisie des marchands et des trafiquants, épousait Marie-Catherine TrottierDesauniers. $^{24}$ Son frère, Joseph Charest, épousait Marguerite Trottier-Desauniers. C'est la famille qui était aussi alliée aux Boucher par le mariage de Françoise-Claire Charest à Jean Boucher, sieur de Montbrun. ${ }^{25}$ Les Charest étaient les cousins des Desauniers et comptaient parmi eux des beaux-frères et des belles-sœurs par suite d'alliances antérieures. ${ }^{26}$

La famille Charly, engagée, nous l'avons fait remarquer, dans la traite des fourrures, était unie à celles des Charest et des Trottier aussi bien qu'à celles des D'Ailleboust et des Tonnancour. Jean-Baptiste Charly avait épousé Louise-Cathe-

21 Le Jeune, II: 496.

22 Le Jeune, II : 290-291. Voir aussi la note suivante.

23 Le Jeune, II: 344.

24 P.-G. Roy, Inventaire des contrats de mariage du régime français conservés aux archives judiciaires de Québec (6 vol., Québec, 1937-1938), Boucault de Godefus, II : 16 et Boucault de Godefus, VI: 58. (Cité désormais sous Roy, Mariage.)

25 Le Jeune, II : 290-291.

26 "Etienne Charest", APC, Dossier personnel, Série E, vol. 72: 5. Voir aussi la section sur la famille Trottier, plus bas. 
rine d'Ailleboust de Manthet. ${ }^{27}$. Louis-Charles de Saint-Ange Charly épousa Anne-Ursule, la fille de René Godefroy, sieur de Tonnancour. ${ }^{28}$ Cette dernière famille était connue dans l'administration, le commerce et la propriété terrienne, particulièrement dans la région des Trois-Rivières. Jacques Charly, le dernier mentionné, épousa Thérèse Charest. ${ }^{29}$

François-Étienne Cugnet, l'homme qui tente de dominer la vie économique de la Nouvelle-France dans la période que nous étudions, avait épousé Louise-Madeleine Dusantoy..$^{30} \mathrm{Cu}$ gnet était le principal fonctionnaire du Domaine d'Occident. Son beau-frère en était un autre. ${ }^{31}$ Sa fille, nous l'avons vu, avait épousé un de Beaujeu. ${ }^{32}$ Le fils de François-Étienne, François-Joseph Cugnet (1720-1789), jurisconsulte bien connu dans la période d'après la Conquête, a épousé en 1741, MarieJoseph, fille de Jacques Belcour de Lafontaine. De Lafontaine était marchand, seigneur, conseiller et entrepreneur en pêcheries; il entretenait aussi des relations commerciales avec les marchands et les administrateurs à Louisbourg. ${ }^{33}$ Cet homme, François-Étienne Cugnet, mieux que tout autre, illustrait ce grand mélange des professions et des fonctions dans la colonie. Il était fonctionnaire et occupait plusieurs emplois: au Conseil Supérieur et au Domaine d'Occident. C'était le membre le plus important de la société qui entreprit d'exploiter les ressources minérales de la région des Trois-Rivières. Il fournissait des marchandises à l'État. Il était seigneur et possédait des propriétés. Il était protégé de l'intendant Hocquart. En même

27 Le Jeune, II: 363-364. Voir aussi la section sur la famille d'Ailleboust, plus bas.

${ }^{28}$ Le Jeune, II: 721. Voir aussi la famille Tonnancour, plus bas.

29 Roy, Mariage, "Hiché", II : 16.

30 Le Jeune, I: 453.

31 Une partie de la section traitant de l'histoire économique est basée sur un article préparé par l'auteur. Il sera bientôt publié sous le titre Inventaire des dépenses générales de la Nouvelle-France, Série $\mathrm{C} 11 \mathrm{~A}$, vols. 113-119, manuscrits, $247 \mathrm{pp}$. L'auteur a préparé de même une collection de documents sur les activités économiques de François-Etienne Cugnet. Elles sont publiées sous le titre "La Banqueroute de François-Etienne Cugnet, 1742", L'Actualité économique, vol. 41, nos 1, 2, 3, 4, 1965-66. 32 Le Jeune, I: 453.

${ }^{33}$ Le Jeune, I: 453-454. 
temps, il était mêlé à d'autres administrateurs et à des personnages profondément intéressés dans tous les secteurs du commerce de la colonie. ${ }^{34}$

Les membres de la famille Douville était très actifs à l'intérieur, aussi bien comme trafiquants que comme commandants de poste. Trois frères participaient au commerce des fourrures, soit comme administrateurs de postes, ou comme fournisseurs. C'était Philippe Dagneau Douville, sieur de la Chaussé; César Dagneau, sieur de Quindre, et Alexandre Dagneau. En plus d'être frères, ils étaient aussi alliés à d'autres familles, qui étaient de même, il fallait s'y attendre, engagés dans la traite des fourrures de l'intérieur.

Philippe Dagneau Douville, sieur de la Chaussé, était marié à Marianne Jarret de Verchères. ${ }^{35}$ César Dagneau, sieur de Quindre, avait épousé Marie-Anne Picoté de Belestre. ${ }^{36}$ Alexandre Douville Dagneau était le mari de Marie Coulon de Villiers. ${ }^{37}$ Il était aussi le beau-frère de François Duplessis Fabert. ${ }^{38}$ Son beau-père et son beau-frère étaient tous les deux commandants de postes à l'intérieur. Une sœur, Marie-Claire, était entrée dans la famille de Saint-Ours, une famille de propriétaires terriens. Son mari était Pierre de Saint-Ours. ${ }^{39}$

La famille de d'Ailleboust, tout comme celle de Dagneau Douville, s'intéressait en tout premier lieu au commerce de la fourrure. Cependant, ce clan se distinguait aussi par ses propriétés terriennes. Marie-Catherine, la fille de Jean-Baptiste d'Ailleboust des Musseaux (1666-1730), avait épousé René Godefroy, sieur de Linctot. ${ }^{40}$ Un fils de Jean-Baptiste, NicolasMarie, s'était marié à un membre de la famille Trottier: Marie-

34 Les renseignements sur les achats faits par l'Etat sont tirés de l'article dont il était question plus haut.

35 Roy, Seigneurie, III: 32.

36 Le Jeune, I: 531.

37 Le Jeune, I: 531.

38 Le Jeune II: 794-795.

39 Le Jeune, I : 531. Voir aussi Saint-Ours, plus bas.

40 Le Jeune, II: 156. 
Louise Trottier Desrivières. ${ }^{41}$ Louise-Charlotte d'Ailleboust Dargenteuil s'était mariée à Pierre Margane, sieur des Forêts de Lavaltrie (1678-1756). La sœur de Lavaltrie, MadeleineLouise, à son tour, avait épousé Paul d'Ailleboust de Perigny. ${ }^{42}$ La branche de Manthet de la famille d'Ailleboust était alliée aux Noyan. Catherine d'Ailleboust de Manthet avait épousé PierreJacques Chavoy de Noyan ${ }^{43}$. C'était le second mariage de Catherine. Elle avait d'abord épousé Jean-Baptiste Charly. ${ }^{44}$ Marie-Madeleine d'Ailleboust de Manthet est donnée dans la liste des congés comme étant l'épouse de Jean-Baptiste Jarret, sieur de Verchères (1687-1752).45 Cette union affermissait les liens de famille non seulement entre les d'Ailleboust et les Verchères, mais encore entre les Douville et les Villiers. Les filles de ce dernier avaient épousé un Douville et François Duplessis Fabert. ${ }^{46}$

La famille des Damours, plus importante dans les premiers temps de la Nouvelle-France, occupait encore une position enviable dans la colonie dans la période 1729-1748, toutefois moindre que dans la période du début. Ils s'intéressaient avant tout à la propriété terrienne et au commerce des fourrures. Mathieu Damours de Clignancourt avait épousé Madeleine Guyon Desprez. ${ }^{47}$ La famille de cette dernière était composée de marchands très importants, fournisseurs de l'Etat pour une grande partie de la viande nécessaire dans le district de Montréal. Madeleine Damours de Plaine en se mariant à Jacques DouaireBondy était entrée dans une autre famille bourgeoise notable. ${ }^{48}$ Les Bondy vendaient à l'Etat, tout comme les Desprez, et étaient aussi engagés dans le commerce des fourrures.

41 Le Jeune, II: 331.

42 Le Jeune, II: 111.

43 Roy, Seigneurie, V: 245.

44 Le Jeune, II : 363-364.

45 E.-Z. Massicotte, "Répertoire des Engagements pour l'Ouest Conservés dans les Archives Judiciaires de Montréal...", RAPQ (1928-30): 277, et Le Jeune, II: 772 .

46 Le Jeune, II : 794-795.

47 Roy, Greffes, "Guillet de Chaumont", XVI: 48.

48 Roy, Mariage, "Barbel", II: 119. Voir aussi Nish, Inventaire, re Desprez et Bondy. 
On voit encore la complexité des relations familiales dans la famille Denys. Ses membres engagés dans la propriété terrienne, l'intérieur, les ventes à l'État, recevaient aussi des subventions de l'État. Louis Denys de la Ronde (1675-1741), avait épousé Louise Chartier de Lotbinière. ${ }^{49}$ Charlotte Denys de la Ronde était la mère de Jean-Baptiste-Nicolas Roch de Ramezay. ${ }^{\text {so }}$ Il s'ensuit que le père était l'aîné Ramezay qui avait été gouverneur de Montréal. Le fils, à son tour, fut un commandant de poste, un administrateur et un seigneur. Eustache Chartier de Lotbinière (1688-1749), seigneur, commerçant de bois, administrateur et membre du Conseil Supérieur, avait épousé la fille de l'ingénieur de la NouvelleFrance, Louise Chaussegros de Lery. ${ }^{51}$ Angélique Chartier de Lotbinière avait épousé le fils de Mathurin-François Martin de Lino. ${ }^{52}$ Le sieur de Lino était marchand, seigneur, membre du Conseil Supérieur et premier conseiller. Avec les mariages de la famille Denys, nous voyons s'étendre les liens entre les Denys, les Lotbinière, les Ramezay, les de Lery, les de Lino.

François Foucault (1690-1766), marchand, administrateur et seigneur, était entré dans la famille de Nafrechoux, une famille considérable dans la vie économique. Michelle-Elisabeth, comme nous l'avons vu, a épousé un de Beaujeu en 1737. Une autre fille, Marie-Claude-Geneviève, avait épousé Guillaume Guillemin en 1744. ${ }^{53}$ Les Guillemin, père et fils, s'appliquaient au commerce, possédaient des terres et s'occupaient d'administration. ${ }^{54}$ Les Guillemin et les Foucault étaient membres du Conseil Supérieur.

Les Gamelin, marchands et seigneurs, étaient alliés aux trafiquants de fourrures et à la bourgeoisie seigneuriale. Ignace Gamelin avait pris pour épouse Marie-Louise Dufrost de la

49 Roy, Seigneurie, III : 80.

50 Roy, Officiers, 213.

51 "Actes de Foi et Hommages", III (1733-1781), APC, "Documents relatifs à la province de Québec: A: Documents généraux, 331.

52 Roy, Seigneurie, III: 80.

53 Le Jeune, I: 642-643.

54 Voir la famille Guillimin ou Guillemin plus bas. 
Jemmerais. Ce dernier était l'héritier de Renée Gaultier de Varennes. ${ }^{55}$ Nous avons vu précédemment que Marie-Clémence Gamelin-Maugras faisait partie de la famille Boucher par son mariage à Joseph Boucher de la Broquerie. ${ }^{56}$ Les GamelinMaugras étaient aussi unis à la famille LaVérendrye. ${ }^{57}$

Foucault, comme nous l'avons vu, était marié à une Nafrechoux. Ainsi en était-il de François de Gannes de Falaise (1677-1746). Il était membre de la bourgeoisie de la fourrure et de l'administration. Sa femme était Marguerite Nafrechoux, “... fille de sieur Isaac Nafrechoux, marchand bourgeois...”. Leur contrat de mariage révèle les bénéfices qui pouvaient être retirés d'un mariage entre ces deux familles. Deux maisons évaluées l'une à 8,000 et l'autre à 18,000 livres. Le fiancé apportait de plus une propriété évaluée à 8,500 livres et celle de l'épouse à 4,000 livres en plus des deux maisons. ${ }^{58} \mathrm{Ce}$ ne sont toutefois pas seulement les arrangements pécuniers qui sont intéressants. Ce sont plutôt les liens maritaux qu'ils révèlent. Les familles des Nafrechoux, des Foucault, des Beaujeu et des Guillemin, mêlées à toutes les activités économiques et politiques importantes de la colonie, constituaient une union personnelle et maritale.

Joseph Fleury de la Gorgendière (1676-1755), comme $\mathrm{Cu}$ gnet, l'un des principaux marchands de la colonie et seigneur, représentait la Compagnie des Indes Occidentales, important fournisseur de l'État. Il avait épousé la fille de Louis Jolliet, Claire. ${ }^{59}$ Sa sœur, en premier lieu épouse de Le Verrier, s'était mariée une seconde fois et son mari était le second marquis de Vaudreuil. ${ }^{60}$ Les deux filles de Joseph Fleury firent aussi des mariages remarquables: Marie-Claire à Thomas-Jacques

55 Roy, Seigneurie, V: 224.

56 Roy, Seigneurie, V: 224.

57 Voir La Vérendrye, plus bas.

58 Contrat de mariage de François de Gannes de Falaise, capitaine d'une compagnie des troupes du détachement de la marine et de Marguerite Nafrechous, Montréal, 29 novembre 1713", APC, "Documents relatifs à la province de Québec: C: District de Montréal, Greffe".

59 Le Jeune, II : 704.

${ }^{60}$ Le Jeune, I: 500 . 
Taschereau, le représentant du trésor de la marine en NouvelleFrance, et Louise-Thérèse à Rigaud de Vaudreuil, le frère du futur gouverneur de la Nouvelle-France, lui-même seigneur et administrateur. ${ }^{81}$ Une autre femme de la famille de la Gorgendière, Marie-Thomas, nièce du Marquis de Vaudreuil, a épousé Thomas-Ignace Trottier Duffy Desauniers. ${ }^{62}$ Le beaupère de Marie-Thomas avait épousé Catherine Charest. ${ }^{63}$ Charlotte, fille de Joseph Fleury de la Gorgendière, sieur Deschambault (1709-1789), avait épousé Joseph Lamarque de Marin, un homme, qui, avec son fils, commandait des postes de l'intérieur. ${ }^{64}$ C'est cette trame de liens maritaux, commerciaux et politiques qui nous force à nous interroger sur le commentaire de Guy Frégault touchant la pénurie de fonds disponibles à l'épouse de Vaudreuil, comme il en est question dans Le grand Marquis. De tels liens maritaux découle une possibilité de profits économiques. ${ }^{65}$

La famille de Charles Guillemin (1676-1739) et celle de son fils Guillaume (1713-1771) étaient des marchands et des fonctionnaires importants de la colonie. Le fils, comme on l'a noté, avait épousé la fille de François Foucault. Sa sœur avait fait un mariage dans cette classe qu'on appelle incorrectement l'aristocratie militaire ou seigneuriale, et que nous appellerons ici la bourgeoisie. Marie-Joseph avait d'abord épousé Jacques Le Gardeur de Saint-Pierre. ${ }^{68}$ Son second mari était Louis-Luc La Corne. ${ }^{67}$ L'autre fille de Charles, Geneviève-Élisabeth s'était mariée à Denis-Charles Regnard Duplessis de Morampont. ${ }^{68}$

François Hazeur (1638-1708), marchand, conseiller et seigneur, avait des enfants qui occupaient des postes dans l'ad-

61 Le Jeune, I: 500.

62 Bonnault, 440, et Roy, Mariage, "Barolet", VI: 58.

63 P.G.R., "Thomas-Ignace Trottier Desaunier (sic)", BRH, vol. 24, no 12 (décembre 1919) : 379-380.

64 Roy, Mariage, "Barolet", II: 80.

65 Guy Frégault, Le grand Marquis (Montréal, Fides, 1952), 116.

66 Roy, Mariage, "Boisseau", III: 172.

67 Le Jeune, II: 727.

68 Roy, Mariage, "Boisseau", III : 172. 
ministration de l'Église et un gendre d'une certaine réputation, Michel Sarrazin. Jean-François Hazeur (1678-1723), conseiller, mourut plutôt jeune. Sa sœur Marie-Ursule épousa Michel Sarrazin, administrateur, seigneur, bénéficiaire de subventions d'État et aussi fournisseur de l'État. Ses beaux-frères, les fils de François, étaient membres du clergé. Pierre Hazeur, sieur de l'Orme, était le représentant du Chapitre de Québec en France. Joseph Thierry, l'autre fils, demeura en Nouvelle-France et occupa des postes importants au Chapitre. Toute la famille était engagée dans une multitude d'activités, soit politiques, religieuses et souvent économiques. ${ }^{69}$

Les Hervieux, une autre famille remarquable dans le commerce de l'intérieur, était liée au trafic des fourrures et à la bourgeoisie seigneuriale. Marie-Anne Hervieux avait épousé Jean-Baptiste Hertel de Rouville. ${ }^{70}$ Marie-Michelle Hervieux avait épousé François-Josué Dubrouil La Corne (1710-1753) . ${ }^{71}$ Luc La Corne, sieur de Chaptes, époux de Marie-Anne Hervieux par un premier mariage, ${ }^{72}$ avait convolé en secondes noces Marie Guillemin.

Henry Hiché, notaire, administrateur et seigneur, s'était marié plusieurs fois. Un de ses mariages fut avec Marguerite Le Gardeur, ${ }^{73}$ et semble avoir été une union fort profitable. Il reçut, comme partie de la dot, la seigneurie de Kamouraska. En 1723, cette terre fut vendue 15,000 livres. ${ }^{74} \mathrm{La}$ fille de Hiché, Marie-Madeleine, a épousé Jean-Baptiste-Ignace Perhuis, marchand, seigneur et administrateur. Charles Perhuis, le père de Jean-Baptiste-Ignace, était aussi un marchand important. ${ }^{75}$

Nicolas Lanoullier, le perpétuel insolvable, administrateur qui menait grand train, a épousé Jeanne-Catherine de Leigne, la

69 Le Jeune, II: 615.

70 Roy, Seigneurie, IV : 78.

71 Le Jeune, II: 21.

72 Le Jeune, II : 21.

73 Roy, Mariage, "Chambalon", IV : 100.

74 J.-B. Gareau, "La Prévôté de Québec-Ses officiers-Ses Registres", RAPQ (1943-44) : 109-11.

75 Gareau : 110-111. 
fille d'un officier de justice de Montréal. ${ }^{76}$ Lanoullier était aussi allié, par les liens du mariage, à la famille Rouville, qui comptait parmi ses membres des commandants de postes et des seigneurs. ${ }^{77}$

Pierre Gaultier de Varennes, sieur de La Vérendrye, était le fils de René Gaultier de Varennes, un gouverneur des TroisRivières. Sa mère était Marie Boucher, fille de Pierre Boucher, aussi gouverneur de la même ville. ${ }^{78}$ Il était apparenté à la famille Gamelin-Maugras. ${ }^{79}$

René Godefroy, sieur de Linctot (1675-1748), s'est marié deux fois. La première fois à une fille de Jean Le Moyne, et la seconde à Marie-Catherine, fille de Jean-Baptiste d'Ailleboust des Musseaux. ${ }^{80}$ Le mariage de son fils révèle quelques-unes des relations économiques et maritales possibles. Il existe un “. . . recu de Louis Linctot à $\mathrm{Sr}$ Blondeau de 4000 livres a compte sur 10,000 prêtées en son contrat de marriage en date du 15 décembre dernier". ${ }^{81}$ Louis-René Godefroy, sieur de Linctot (1709-1745), avait épousé Apolline Blondeau. ${ }^{82}$

Une enquête sur le mariage de Paul-Louis Lusignan, commandant de poste, apporte une autre illustration du mariage comme moyen de puissance, de position et de richesse. Lusignan était marié à Madeleine Bouat. ${ }^{83}$ Il était le beau-frère de Jean Poulin de Courval, ${ }^{84}$ marchand. Le beau-père de Lusignan était François-Marie Bouat, administrateur et seigneur de Terrebonne, la seigneurie qui devait être vendue plus tard à l'abbé Lepage. La seconde épouse de Bouat était Agathe Le Gardeur de Repentigny. Les filles de Bouat avaient contracté mariage en

76 Gareau: 74-78, et Roy, Mariage, "Barbel", I: 32. Le Jeune, II: 66, fait erreur lorsqu'il tente d'identifier l'épouse de Lanoullier.

77 Gareau: 74-78.

78 Gosselin, II: 24, et Roy, Officiers, 235.

79 Le Jeune, II : 42-43.

80 Le Jeune, II: 156.

81 Roy, Greffes, “G. J. Porlier", XV: 71.

82 Le Jeune, II: 156.

83 Le Jeune, II: 195.

84 Le Jeune, I: 210. 
des familles, comme nous l'avons vu souvent, fort mêlées à une variété d'occupations dans la colonie. Louise-Jeanne avait épousé François Daine, administrateur, marchand et seigneur. Madeleine-Thérèse s'était mariée à Jean Poulin de Courval en premier lieu et ensuite à Jean-Baptiste de Gannes Falaise. Un fils de ce mariage, François, épousa Marie-Jeanne Gaultier de Varennes ${ }^{85}$. En commençant avec les Lusignan nous voyons la ligne s'étendre ainsi: Lusignan-Bouat-Courval-Daine-de Gannes-Varennes: terre, commerce de fourrure, seigneuries, administration et commerce.

Pierre-Thomas Tarieu de Lanaudière, parfois connu sous le nom de la Pérade (1677-1757), appartenait surtout au monde militaire et au commerce des fourrures. Il épousa MarieMadeleine Verchères. Sa sœur, Marguerite-Marie-Anne, épousa Richard Testu de la Richardière, le capitaine du port de Québec et une figure importante dans ie transport et le commerce. Marguerite épousa en second mariage Antoine Coulon, sieur de Villiers. ${ }^{86}$

Jean-Baptiste Neveu, sieur de la Bretonnière, était un marchand notable dans la colonie. Il était fournisseur à l'état et trafiquant de fourrures. Sa fille Marie avait épousé Hector Rouer de Villeray, administrateur et seigneur. ${ }^{87}$ Jean-François Neveu, le fils de Jean-Baptiste, avait épousé Charlotte-Ursule Boucher en 1737. ${ }^{88}$

Pierre-Jacques Chavoy de Noyan, qui avait d'abord épousé Catherine d'Ailleboust de Manthet, ${ }^{89}$ était aussi notable pour d'autres mariages. L'union antérieure de sa femme avait été à Jean-Baptiste Charly. ${ }^{90}$ Noyant était aussi allié aux Le Moyne, le

85 Le Jeune, I: 210.

86 Le Jeune, II : 57.

87 Roy, Greffes, “Guillet de Chaumont", XVI : 118, 143-44.

88 "Contrat de mariage du Sr. Jean Francois Neveu et Demoiselle Charlotte Boucher, Le 5 juin 1737", APC, "Papiers d'avant la conquête: $\mathrm{H}$ : Boucher de Boucherville".

89 Roy, Seigneurie, V : 245.

90 Le Jeune, II : 363-364. 
premier Baron était son oncle. ${ }^{91}$ Sans trop approfondir les relations maritales des Le Moyne, remarquons cependant quelques liens. René Godefroy, sieur de Linctot, nous l'avons dit, avait épousé une fille de Jean Le Moyne. ${ }^{92}$ Jean Bouillet de la Chassaigne, gouverneur de Montréal et des Trois-Rivières, était le beau-frère du Baron de Longueuil. ${ }^{93} \mathrm{Il}$ avait épousé Marie-Anne Le Moyne. ${ }^{94}$ Paul-Joseph Le Moyne, le chevalier de Longueuil, membre de la bourgeoisie de la fourrure et administrateur, avait épousé Marie-Geneviève de Joybert. ${ }^{95}$ Les Joybert étaient apparentés aux Vaudreuil parce que le premier Marquis avait épousé Louise-Élisabeth Joybert. ${ }^{96}$ Les liens sont les suivants: Noyan-d'Ailleboust-Charly-Le Moyne-de la Chassaigne-Vaudreuil. Cette liste plutôt impressionnante inclut des gouverneurs des Trois-Rivières, de Montréal et de la Nouvelle-France, des commandants de postes, des trafiquants de fourrures, des gens intéressés à la propriété terrienne et au commerce en général.

Nicolas-Marie d'Avènes, sieur de Méloizes (1696-1743), était commandant de poste, administrateur et bénéficiait de subventions de l'état. Il était marié à Angélique Chartier de Lotbinière. Leur fille, Angélique, on pourrait dire leur fille célèbre, ${ }^{97}$ avait épousé Michel-Jacques-Hughes Péan. ${ }^{98}$ Le père de Péan était Jacques-Hughes-Péan de Livaudière (1682-1747). Le père, tout comme le fils, occupait une variété de postes et

91 "Ministre au Baron de Longueuil, Compiègnes, 2 mai 1729", APC, B, vol. 53-2, f 535 .

92 Le Jeune, II : 156.

93 Benjamin Sulte, "Les Gouverneurs des Trois-Rivières", 70-71.

94 Le Jeune, II: 14.

95 L'abbé Azarie Couillard-Després, La première famille française au Canada, ses alliés et ses descendants (Montréal: Imprimerie de l'Ecole Catholique des Sourds Muets, 1906), 224.

96 P.-G. Roy, La ville de Québec sous le régime français (Québec: Rédempti Paradis, 1930) II: 25, 430-431. Voir aussi Nish, "Une bourgeoisie coloniale".

97 Voir Guy Frégault, Frangois Bigot, administrateur francais, (2 vol., Montréal: Les Etudes de l'Institut d'Histoire de l'Amérique Française, 1948), 19.

88 Le Jeune, I : 506. 
de fonctions: trafiquant de fourrures, administrateur, fournisseur à l'État et seigneur. ${ }^{99}$

Jean-Baptiste Pommereau, marchand et administrateur, était aussi fournisseur à l'État. Il était marié à une Boucher; Françoise Boucher était la fille de Pierre Boucher de Boucherville et de Charlotte Denys. ${ }^{100}$ Pierre Raimbault avait les mêmes occupations que Pommereau. Sa seconde épouse était Louise Nafrechoux. Sa fille, Marguerite, maria un membre de la famille de Boucherville. ${ }^{101}$ Une autre Raimbault, Marie-LouiseCatherine, épousa Julien Trottier Desrivières, ces derniers étaient une branche de la famille des Trottier, et était alliée aux Trottier Duffy Desauniers et aux Trottier des Ruisseaux. ${ }^{102}$

Étienne Rocbert, et son fils, détenaient le monopole du poste de garde-magasin à Montréal durant la période que nous étudions. Ils étaient aussi, par mariage, alliés aux Varennes, aux Bégon et à Honoré Michel, le commissaire de la marine à Montréal. Ce dernier, Honoré Michel de Villebois, sieur de la Rouvillière, "conseiller du Roi, commissaire de la Marine, ordonnateur et subdélégué à l'intendance du Canada...", était marié à Marie-Catherine-Élisabeth Bégon, la fille de ClaudeMichel Bégon et de Marie-Élisabeth Rocbert. ${ }^{103}$ Étienne Rocbert, le fils, était marié à Marguerite-Barbe de Puygibault, dont la mère était Marguerite de Varennes. ${ }^{104}$ Les liens maritaux, dans ce cas, entraînent alliance avec le chef de l'administration civile à Montréal, un gouverneur des Trois-Rivières qui était le frère d'un intendant de la colonie, famille largement adonnée à la traite des fourrures et une famille qui possédait les postes les plus importants pour ce qui est des fournitures à l'État.

${ }^{99}$ Le Jeune, I: 507.

100 Roy, Seigneurie, II : 279.

101 Le Jeune, II : 496.

102 Roy, Greffes, "G. J. Porlier", XV : 65.

103 Roy, Greffes, "Guillet de Chaumont", XVI: 171-172. Voir aussi Frégault, Bigot, I: 343, n. 16.

104 Roy, Greffes, "Guillet de Chaumont", XVI: 171-172. 
Pierre, le chevalier de Saint-Ours (1673-1750), appartenait à une branche de la famille Deschaillon. Les membres de la famille étaient des commandants de postes importants, des seigneurs et des administrateurs. Le premier mariage de Pierre se fit avec un membre de la famille de Céloron de Blainville. Son second mariage fut avec Marie-Claire Dagneau. ${ }^{105}$ Son neveu, Pierre-Roch de Saint-Ours (1712-1782), était marié à Louise-Charlotte Deschamps de Boishébert. ${ }^{106}$ Cette dernière famille occupait des postes à l'intérieur et dans l'administration et possédait des seigneuries.

Michel Sarrazin, que nous avons mentionné comme étant allié aux Hazeur, l'était aussi aux Varennes. Charlotte-Louise, en 1746, épousait Jean-Hippolyte Gaultier de Varennes. ${ }^{107}$ Sarrazin, alors, devenait le beau-père de Jean-Hippolyte. Les Varennes, nous nous en souvenons, étaient alliés aux Boucher, et les Boucher à presque tous les autres.

Jean-Pascal Taché (1697-1788) était marchand en Nouvelle-France. Sa femme était Marie-Anne Jolliet. ${ }^{108}$ Claire Jolliet, nous l'avons dit, avait épousé Joseph Fleury de la Gorgendière. De la Gorgendière était le beau-père de ThomasJacques Taschereau et de Rigaud de Vaudreuil, et le beau-frère du Marquis de Vaudreuil. ${ }^{109}$

Les de Tonnancour des Trois-Rivières, marchands importants, administrateurs et seigneurs, étaient alliés à la famille Charly de Montréal ${ }^{110}$ et aux Ramezay. Louise Godefroy était la femme de Jean-Baptiste-Nicolas Roch de Ramezay. ${ }^{111}$

Les Trottier se subdivisaient en plusieurs branches, qui sont, franchement, très difficiles à séparer. Dans les documents

105 Le Jeune, II : 599.

106 Le Jeune, II : 600.

${ }^{107}$ Le Jeune, II: 615, et Roy, Mariage, "Dulaurent”, II: 65.

108. Le Jeune, II: 685.

109 Le Jeune, II: 697. Voir aussi : "La Famille Tachereau, Généalogie", $A P C$, "La famille Tachereau: Papiers d'avant la conquête".

110 Le Jeune, II: 721.

111 Le Jeune, II : 499. 
de la période, on réfère à eux comme étant les Duffy, Desauniers, Duffy Desauniers, Trottier Desrivières, Trottier Desruisseaux et Trottier Duffy Desauniers. Les mariages avaient produit une grande complexité de relations. Pierre Trottier Desauniers, partenaire de François Martel de Brouage dans l'industrie de la pêche, s'était marié à Catherine Charest. Son fils portait le nom de Thomas-Ignace Trottier Duffy Desauniers. Les Duffy venaient des Charest. Le fils Thomas-Ignace, s'était marié, nous l'avons dit déjà, à Marie-Thomas Fleury de la Gorgendière. ${ }^{112}$ Etienne Trottier Desauniers, à son tour, avait épousé la fille de Bernard Damours de Plaine, sieur de la Freneuse. ${ }^{113}$

Julien Trottier Desrivières avait épousé Marie-Louise Catherine Raimbault. ${ }^{114}$ Marie-Louise Trottier Desrivières s'était mariée à d'Ailleboust de Musseaux. ${ }^{115}$ Marie-Catherine Trottier Desruisseaux est donnée comme la veuve de François-Marie Picoté de Belestre. 116 Une autre Belestre avait épousé César Dagneau. La famille Trottier, par elle-même, ou par ses relations, participait à toutes les fonctions de la vie commerciale et politique de la colonie.

Que révèle cette enquête sur les liens établis par le mariage ? Est-il vrai, comme Charlevoix l'a écrit, qu'il y a une classe de “... gentilshommes et d'officiers" ?117 Existait-il, comme il est écrit dans le mémoire attribué à Hocquart, une classe noble et une classe d'officiers distincte de la classe seigneuriale et de la classe des marchands ? ${ }^{118}$ C'est fort douteux. Ce qui est très

112 Bonnault, 440, et Roy, Mariage, "Barolet", VI: 58. Voir aussi P.G.R., "Thomas Ignace Trottier Desaunier (sic)", 379-380.

118 Le Jeune, I: 466, et Roy, Mariage, "Boucault de Godefus", VI: 58.

114 "Extrait des Registres des baptêmes, mariages et sépultures, fait dans la paroisse de Montréal, sous le titre de S. Nom de Marie, dans l'Ile, comté et district de Montréal . . . pour l'année mil sept cent quarante huit", APC, "Papiers d"avant la conquête: H: La famille Testard de Montigny".

115 Le Jeune, II: 331.

116 F.-J. Audet, "Les Législateurs de la Province de Québec", BRH, 31, no 12 (décembre 1925) : 537. 252-254.

117 F.-X. Charlevoix, Histoire de la Nouvelle-France, V: 117-118,

118 Nish, Documents, 180. 
évident, c'est que dans la Nouvelle-France, les liens matrimoniaux s'établissent selon le même modèle que dans les colonies angloaméricaines. La mobilité sociale existait à un degré frappant. Il ne s'agissait pas cependant d'une mobilité verticale, c'est-à-dire qu'un habitan, dans le sens péjoratif du mot, pouvait monter de rang. Il s'agissait plutôt d'une mobilité horizontale qui tendait à effacer les démarcations de classes entre les gens impliqués dans des fonctions purement économiques qui forment généralement la classe appelée bourgeoisie, et ceux qui appartenaient à la noblesse ou à l'aristocratie, habituellement donnée comme la partie essentielle de l'Ancien Régime. Les familles importantes de la colonie se mariaient entre elles. Elles réunissaient tous les privilèges importants que pouvaient leur obtenir le pouvoir politique et le pouvoir économique. Les marchands, les trafiquants de fourrures, les seigneurs et les administrateurs, en raison au moins des liens de mariage, rendent impossible la répartition des classes sociales de la société canadienne selon le concept classique. Il n'existait pas de premier ou de second ou de tiers État, dans le sens général de ces termes.

CAMERON NISH

\section{APPENDICE}

Le tableau suivant permet de mieux saisir la complexité et peut-être le sens des liens de mariage. Nous avons choisi quelques-unes des familles dont les noms apparaissent dans cet essai, et nous avons dressé une liste selon les liens étroits qui les unissaient, et une autre selon les liens moins étroits. Dans deux cas, celui des d'Ailleboust et des Boucher, les deux listes apparaissent. Les liens indiquent ceux de père, fils, fille, de beau-père ou de beau-frère. Les autres degrés de parenté sont indiqués dans les liens éloignés. Nous présumons qu'il était possible qu'il existât des liens politiques, sociaux et économiques, même dans des cas d'affiliation lointaine.

\section{d'Ailleboust}

Parenté proche: de Manthet, de la Madeleine, Périgny, des Musseaux, Linctot, Trottier, Lavaltrie, Noyan, Charly, Verchères, Douville, Villiers, Duplessis-Faber. 
Parenté éloignée: Trottier, Neveu, Boucher, Gamelin-Maugras, Sabrevois, Sabrevois de Bleury, La Vérendrye, Raimbault, Charest, de Tonnancour, Charly, Linctot, Lavaltrie, Verchères, Noyan, Dagneau-Douville, Villiers, Duplessis-Faber, Blondeau, Saint-Ours, Boishébert, Blainville.

\section{Boucher}

Parenté proche: Gamelin-Maugras, Sabrevois, La Vérendrye, Raimbault, Charest, Neveu.

Parenté éloignée: Gamelin-Maugras, Sabrevois, Sabrevois de Bleury, La Vérendrye, Raimbault, Charest, Neveu, Trottier, Charly, d'Ailleboust, de Tonnancour, Varennes, Pommereau, Denys.

\section{Beaujeu}

Parenté proche: Cugnet, Varin, Foucault, Charly.

$$
\text { Dạgnéâu-Doưuiville }
$$

Parenté proche: Verchères, Belestre, Villiers, Duplessis-Faber, Saint-Ours.

\section{Charly}

Parenté éloignée: Trottier, Neveu, Boucher, Gamelin-Maugras, Sabrevois, Sabrevois de Bleury, La Vérendrye, Raimbault, Charest, d'Ailleboust, de Tonnancour.

\section{Denys}

Parenté proche: de Lotbinière, Ramezay, de Lety, de Lino, Boucher.

\section{Foucault}

Parenté éloignée: Beaujeu, Cugnet, Varin, Charly, Nafrechoux, Guillemin, de Gannes. de la Gorgendière

Parenté proche: Jolliet, Vaudreuil, Taschereau, Trottier, Marin.

$$
\text { Guillemin ou Guillimin }
$$

Parenté proche: Foucault, LeGardeur de Saint-Pierre, La Corne, Duplessis de Morampont. 
Noyan

Parenté proche: d'Ailleboust, Le Moyne, Linctot, de la Chassaigne, Joybert, Vaudreuil.

\section{Raimbault}

Parenté éloignée: Boucher Sabrevois, Sabrevois de Bleury, La Vérendrye, Gamelin-Maugras, Charest, Neveu, Trottier, Charly, d'Ailleboust, de Tonnancour, Nafrechoux.

\section{Trottier}

Parenté éloignée: Charest, Neveu, Boucher, Gamelin-Maugras, Sabrevois, Sabrevois de Bleury, La Vérendrye, Raimbault, Charly, d'Ailleboust, de Tonnancour, de la Gorgendière, Damours.

On nous prie d'annoncer que l'International Council on Archives tiendra un Congrès spécial à Washington, du 10 au 13 mai 1966. Avis aux intéressés. Prière de communiquer avec M. Ken Munden, Director, Special Projects Staff, National Archives and Records Service, Washington, D.C., 20408. 